\title{
Relativistic corrections to the non-Born-Oppenheimer energies of the lowest singlet Rydberg states of ${ }^{3} \mathrm{He}$ and ${ }^{4} \mathrm{He}$
}

\author{
Monika Stanke \\ Department of Chemistry, University of Arizona, Tucson, Arizona 85721 \\ and Institute of Physics, Nicholas Copernicus University, ul. Grudziadzka 5, PL 87-100 Toruń, Poland \\ Dariusz Kędziera \\ Department of Chemistry, Nicholas Copernicus University, ul. Gagarina 7, PL 87-100 Toruń, Poland
}

Sergiy Bubin

Department of Chemistry, University of Arizona, Tucson, Arizona 85721

Ludwik Adamowicz

Department of Chemistry, University of Arizona, Tucson, Arizona 85721

and Department of Physics, University of Arizona, Tucson, Arizona 85721

(Received 21 December 2006; accepted 4 April 2007; published online 17 May 2007)

In this work the authors present an approach to calculate the leading-order relativistic corrections for ground and excited states of helium isotopomers. In the calculations they used variational wave functions expanded in terms of explicitly correlated Gaussians obtained without assuming the Born-Oppenheimer approximation. (C) 2007 American Institute of Physics.

[DOI: $10.1063 / 1.2735305$ ]

\section{INTRODUCTION}

The helium atom is a system that has been described in calculations since the very early stages of the development of quantum mechanics. It is also one of the systems where the experiment has achieved the highest levels of precision. Recent theoretical studies of the helium atom that include the works performed by Morton et al., ${ }^{1}$ Korobov and Yelkhovsky, ${ }^{2}$ Korobov, ${ }^{3}$ and Pachucki, ${ }^{4-6}$ have demonstrated that by systematically including relativistic and QED corrections to the nonrelativistic energies of the ground and excited states of this system, one can achieve an accuracy of the predicted ionization and transition energies that in some cases exceed the accuracy of the present-day experiment. The recently published summary of the available theoretical and experimental results for bound stationary states of He by Morton et al. ${ }^{1}$ demonstrates the high level agreement between theory and experiment very well. It also shows that for a few states such as ${ }^{2} 1 P_{1}$ and ${ }^{2} 3 P_{J}$ there is still some noticeable disagreement between the theory and the experiment. ${ }^{6,7}$

To achieve a high level of agreement between the experiment and the theory for electronic transition energies, the accounting for relativistic corrections that include, apart from the dominant $\alpha^{2}$-dependent terms (where $\alpha=1 / \mathrm{c}$ is the fine structure constant), also QED terms ${ }^{8}$ is needed. Including the latter terms made the QED part of the calculations for the He atom ${ }^{1}$ essentially exact, up to terms of the order of $\alpha^{3}$. Furthermore, terms of the order of $\alpha^{4}$ were included for some lower states from the calculations performed by Korobov and Yelkhovsky, ${ }^{2}$ by Yelkhovsky, ${ }^{9}$ by Pachucki, ${ }^{5}$ and by Drake and Martin. ${ }^{10}$ Moreover, there are works where the QED terms of the order of $\alpha^{6}$ for the $\mathrm{He}$ atom were calculated. $^{4,6}$
The He calculations also included corrections for finite values of the ${ }^{3} \mathrm{He}$ and ${ }^{4} \mathrm{He}$ charge radii of 1.9659 and $1.167 \mathrm{fm}$, respectively, which were derived from the isotope shift measured by Shiner et al. ${ }^{11}$ and from the measurements of the Lamb shift of the muonic hydrogen. ${ }^{11,12}$ The theoretical results included in work Ref. 1 will be used as a reference for the results obtained in this work.

The aim of the present study is to describe the first stage of the development of a computational approach that has the capability of producing results for ground and excited states of atoms and, eventually, for molecular systems with more than two electrons with a similar accuracy as the results obtained for helium. ${ }^{1}$ The most straightforward way of developing such an approach would be by extending the Slatertype or Hylleraas-type basis set used in the calculations reported in the work of Morton et al. to atomic systems with more than three electrons. However, such an extension has not been accomplished yet due to problems with calculating the necessary matrix elements with explicitly correlated Slater- or Hylleraas-type functions. Only calculations with three-electron integrals (or a total of four particles) have been reported thus far. ${ }^{13-18}$ Alternative basis functions that can be used in very accurate atomic calculations are explicitly correlated Gaussian functions. Gaussians are less effective than Slaters in describing the cusp behavior of the wave functions, but their use leads to much easier integrals, which, for a one-center expansion of the wave function, can be analytically calculated using standard procedures for any number of electrons. In recent years we have used various types of Gaussian basis functions in very accurate atomic and molecular calculations. In those calculations we have employed an approach departing from the Born-Oppenheimer (BO) approximation, whose development has been carried out in our research group. ${ }^{19-27}$ If the BO approximation is not assumed, 
the motion of the electrons and the nuclei are treated on equal footing, and since these motions are highly correlated (coupled), one has to use basis functions that explicitly depend on the distances between the particles (nuclei and electrons) in expanding the wave function. The explicitly correlated Gaussians are such functions.

Recently, explicitly correlated Gaussians were used by Pachucki and Komasa ${ }^{28,29}$ to calculate new variational upper bounds to the BO energies of the two lowest $S$ states of the Be atom. They also calculated relativistic and QED energy corrections of the orders of $\alpha^{2}$ and $\alpha^{3}$ for those states. The corrections were determined as expectation values of the corresponding operators using the $\mathrm{BO}$ electronic wave functions. In addition they also calculated corrections due to the finite mass of the nucleus using the perturbation theory approach. Since the corrections are very small, the perturbative approach is capable of providing very accurate values of those corrections. There is an interesting analysis in the work of Pachucki et al. ${ }^{30}$ concerning the accuracy of the relativistic integrals. They compare the direct algorithm of calculating those integrals with an algorithm based on the so-called "Drachmanization" and show that this procedure leads to a much faster convergence of the integral values with the size of the basis set. However, when the basis set size becomes large, the values obtained with the direct procedure and with the procedure based on the Drachmanization are virtually the same. Since in the present calculations we use very extended basis sets, the algorithms we developed are based on the direct approach.

The approach we have been developing is somewhat different from that of Pachucki and Komasa because in our nonrelativistic calculations we do not assume the BO approximation and the finite mass of the nucleus is explicitly included in generating the energy and the wave function. As a result, we obtain different wave functions and different energies for different isotopes. With those wave functions we then calculate expectation values of the relativistic operators. In this way our atomic relativistic corrections include not only the electronic contributions, but also contributions due to the nucleus. In this work we show the application of the approach in calculations of the $\alpha^{2}$-dependent relativistic corrections to the energies of the ground and excited states of the $S$ symmetry of ${ }^{3} \mathrm{He}$ and ${ }^{4} \mathrm{He}$. These corrections include the mass-velocity and Darwin terms, as well as terms due to magnetic orbit-orbit and Fermi contact interactions. The algorithms for the former were derived for a more general case of a diatomic system in our recent works. ${ }^{25-27}$ In the atomic case considered here, we used a simplified version of those algorithms. The algorithms for the latter terms have been derived and implemented in this work.

The calculations of the relativistic effects using the non-BO wave functions allow for a direct calculation of the isotopic energy shifts of such quantities as the ionization potential and transition energies. In this work we show the calculations of those shifts for the ${ }^{3} \mathrm{He}$ and ${ }^{4} \mathrm{He}$ isotopes.

\section{THE METHOD USED IN THE CALCULATIONS}

\section{A. The internal Hamiltonian}

The total nonrelativistic Hamiltonian can be exactly separated into the Hamiltonian representing the kinetic energy of the center-of-mass motion and the internal Hamiltonian. In our approach, this transformation is done by replacing the laboratory coordinate system by a new system whose first three coordinates are the laboratory Cartesian coordinates of the center of mass, $\mathbf{r}_{0}$, and the remaining coordinates are internal Cartesian coordinates. The origin of the internal coordinate system is placed at the nucleus (particle 1 with mass $M_{1}$ called the reference particle). The other particles (electrons) are referred to as the reference particle using the Cartesian position vectors $\mathbf{r}_{i}$. The internal Hamiltonian $H_{\text {int }}$ for the helium atom is

$$
\begin{aligned}
H_{\mathrm{int}}= & -\frac{1}{2}\left(\sum_{i=1}^{2} \frac{1}{\mu_{i}} \nabla_{\mathbf{r}_{i}}^{2}+\sum_{i=1}^{2} \sum_{j \neq i}^{2} \frac{1}{M_{1}} \nabla_{\mathbf{r}_{i}} \cdot \nabla_{\mathbf{r}_{j}}\right) \\
& +\sum_{i=1}^{2} \frac{q_{0} q_{i}}{r_{i}}+\sum_{i=1}^{2} \sum_{i<j}^{2} \frac{q_{i} q_{j}}{r_{i j}} .
\end{aligned}
$$

The internal Hamiltonian [Eq. (1)] describes two pseudoparticles (pseudoelectrons) with charges $q_{i}=-1, i=1,2$, and reduced masses $\mu_{i}=M_{1} M_{i+1} /\left(M_{1}+M_{i+1}\right)$ (where $M_{2}=M_{3}=1$ are electron masses) moving in the central potential of the charge of the reference particle $\left(q_{0}=2\right)$. The motions of the pseudoparticles are coupled through the mass polarization term $\sum_{i=1}^{2} \Sigma_{j \neq i}^{2} 1 / M_{1} \nabla_{\mathbf{r}_{i}} \cdot \nabla_{\mathbf{r}_{j}}$ and through the Coulombic interactions dependent on the distances of the pseudoparticles from the central charge, $r_{i}=\left|\mathbf{r}_{i}\right|$, and their relative distances, $r_{i j}=\left|\mathbf{r}_{j}-\mathbf{r}_{i}\right|$.

\section{B. Relativistic Hamiltonian for many-particle systems}

For small atomic and molecular systems the account of the relativistic and QED effects is usually done using the first-order perturbation theory and calculating those corrections as expectation values of the corresponding operators. The corrections can be grouped into terms, each proportional to a certain power of the fine structure constant $\alpha$,

$$
H=H_{\mathrm{int}}+\alpha^{2} H_{\mathrm{rel}}+\alpha^{3} H_{\mathrm{QED}(3)}+\alpha^{4} H_{\mathrm{QED}(4)}+\cdots,
$$

where $H_{\text {int }}$ is the nonrelativistic Hamiltonian described in the previous section, $H_{\text {rel }}$ includes all relativistic terms whose contributions are proportional to $\alpha^{2}$, and $H_{\mathrm{QED}(3)}$ and $H_{\mathrm{QED}(4)}$ group all contributions proportional to $\alpha^{3}, \alpha^{4}$, etc. We will now discuss the $\alpha^{2}$ contributions in the case of the He atom, where either all particles are fermions and have spins equal to $1 / 2$ (the ${ }^{3} \mathrm{He}$ case), or where two particles have spins equal to $1 / 2$ and the third is a boson ( $\alpha$-particle) with a zero spin (the ${ }^{4} \mathrm{He}$ case).

While a full separation of the laboratory Hamiltonian into the Hamiltonian describing the kinetic energy of the center-of-mass motion and the internal Hamiltonian can be exactly performed, the separation of the relativistic Hamiltonian into the internal and external parts is not exact. In general, the Breit-Pauli Hamiltonian after the transforma- 
tion from the laboratory coordinate system to the new system of coordinates can be written as a sum of three terms,

$$
H_{\mathrm{rel}}=H_{\mathrm{rel}}^{\mathrm{CM}}+H_{\mathrm{rel}}^{\mathrm{int}}+H_{\mathrm{rel}}^{\mathrm{CM}-\mathrm{int}},
$$

where $H_{\mathrm{rel}}^{\mathrm{CM}}$ is the term describing the relativistic effects of the motion of the center of mass, $H_{\mathrm{rel}}^{\text {int }}$ describes the internal relativistic effects, and $H_{\text {rel }}^{\mathrm{CM} \text {-int }}$ describes the relativistic coupling of the internal and external motions. The relativistic corrections to the internal states of the system are calculated using $H_{\mathrm{rel}}^{\mathrm{int}}$. For states with the $S$ symmetry of ${ }^{4} \mathrm{He}$ and ${ }^{3} \mathrm{He}$, the transformation of the coordinate system leads to the internal relativistic operators in the following form:

$$
\begin{aligned}
H_{\text {rel }}^{\text {int }}\left({ }^{4} \mathrm{He}\right)= & -\frac{1}{8}\left[\frac{1}{m_{0}^{3}}\left(\nabla_{1}+\nabla_{2}\right)^{4}+\frac{1}{m_{1}^{3}} \nabla_{1}^{4}+\frac{1}{m_{2}^{3}} \nabla_{2}^{4}\right]-\frac{\pi}{2}\left[\frac{1}{m_{1}^{2}} q_{0} q_{1} \delta^{3}\left(r_{1}\right)+\frac{1}{m_{2}^{2}} q_{0} q_{2} \delta^{3}\left(r_{2}\right)+\left(\frac{1}{m_{1}^{2}}+\frac{1}{m_{2}^{2}}\right) q_{2} q_{1} \delta^{3}\left(\mathbf{r}_{12}\right)\right] \\
& -\frac{1}{2} \frac{q_{0} q_{1}}{m_{0} m_{1}}\left[\frac{1}{r_{1}} \nabla_{1} \cdot \nabla_{1}+\frac{1}{r_{1}^{3}} \mathbf{r}_{1} \cdot\left(\mathbf{r}_{1} \cdot \nabla_{1}\right) \nabla_{1}\right]-\frac{1}{2} \frac{q_{0} q_{2}}{m_{0} m_{2}}\left[\frac{1}{r_{2}} \nabla_{2} \cdot \nabla_{2}+\frac{1}{r_{2}^{3}} \mathbf{r}_{2} \cdot\left(\mathbf{r}_{2} \cdot \nabla_{2}\right) \nabla_{2}\right]-\frac{1}{2} \frac{q_{0} q_{1}}{m_{0} m_{1}}\left[\frac{1}{r_{1}} \nabla_{1} \cdot \nabla_{2}\right. \\
& \left.+\frac{1}{r_{1}^{3}} \mathbf{r}_{1} \cdot\left(\mathbf{r}_{1} \cdot \nabla_{1}\right) \nabla_{2}\right]-\frac{1}{2} \frac{q_{0} q_{2}}{m_{0} m_{2}}\left[\frac{1}{r_{2}} \nabla_{2} \cdot \nabla_{1}+\frac{1}{r_{2}^{3}} \mathbf{r}_{2} \cdot\left(\mathbf{r}_{2} \cdot \nabla_{2}\right) \nabla_{1}\right]+\frac{1}{2} \frac{q_{1} q_{2}}{m_{1} m_{2}}\left[\frac{1}{r_{12}} \nabla_{1} \cdot \nabla_{2}+\frac{1}{r_{12}^{3}} \mathbf{r}_{12} \cdot\left(\mathbf{r}_{12} \cdot \nabla_{1}\right) \nabla_{2}\right] \\
& -\frac{8 \pi}{3}\left[\left(\mathbf{S}_{1} \cdot \mathbf{S}_{2}\right) \delta^{3}\left(\mathbf{r}_{12}\right)\right]
\end{aligned}
$$

and

$$
\left.H_{\mathrm{rel}}^{\mathrm{int}}{ }^{3} \mathrm{He}\right)=H_{\mathrm{rel}}^{\mathrm{int}}\left({ }^{4} \mathrm{He}\right)+\frac{\pi}{2}\left[\frac{1}{m_{0}^{2}} q_{0} q_{1} \delta^{3}\left(\mathbf{r}_{1}\right)+\frac{1}{m_{0}^{2}} q_{0} q_{2} \delta^{3}\left(\mathbf{r}_{2}\right)\right]-\frac{8 \pi}{3}\left[\frac{q_{0} q_{1}}{m_{0} m_{1}}\left(\mathbf{S}_{0} \cdot \mathbf{S}_{1}\right) \delta^{3}\left(\mathbf{r}_{1}\right)+\frac{q_{0} q_{2}}{m_{0} m_{2}}\left(\mathbf{S}_{0} \cdot \mathbf{S}_{2}\right) \delta^{3}\left(\mathbf{r}_{2}\right)\right] .
$$

The difference between the two Hamiltonians $H_{\text {rel }}^{\text {int }}\left({ }^{4} \mathrm{He}\right)$ and $H_{\text {rel }}^{\text {int }}\left({ }^{3} \mathrm{He}\right)$ results from the fact the ${ }^{3} \mathrm{He}$ nucleus is a fermion with spin $\frac{1}{2}$ and the ${ }^{4} \mathrm{He}$ nucleus is a boson with zero spin.

\section{RESULTS}

The general form of the basis function used here to calculate states of the $S$ symmetry for ${ }^{3} \mathrm{He}$ and ${ }^{4} \mathrm{He}$ is $(\otimes$ is the Kronecker product symbol),

$$
\phi_{k}=\exp \left[-\mathbf{r}^{\prime}\left(L_{k} L_{k}^{\prime} \otimes I_{3}\right) \mathbf{r}\right],
$$

where for He, the $\mathbf{r}$ vector is a six-component vector of the internal Cartesian coordinates of the two pseudoparticles, $L_{k}$ is a $2 \times 2$ rank 2 lower triangular matrix of nonlinear variation parameters, and $I_{3}$ is the $3 \times 3$ identity matrix. To ensure the proper permutational symmetry of the two electrons, the appropriate symmetry projections were applied to the basis functions. The wave functions for the ground state and the excited states were obtained using the variational method by minimizing the energy of each state in a separate calculation. The minimization was done with respect to the linear expansion coefficients $c_{k}$ and with respect to the nonlinear parameters of the basis functions, i.e., the basis set exponent matrices $L_{k}$. In the minimization we used analytically calculated gradients of the energy with respect to $\left\{c_{k}\right\}$ and $\left\{L_{k}\right\}$. The use of the analytical gradient was key in obtaining very accurate results in the energy minimization.

1500 Gaussians for each state were used in our calculations. Our previous experience with atomic calculations have shown that it is not necessary to optimize the linear parameters for all the isotopes. Equally accurate results can be obtained by performing nonlinear parameter optimization and generating the basis set for one of the isotopes $\left({ }^{4} \mathrm{He}\right.$ is this work) and using it in the calculations of other isotopes where only the linear parameters are optimized through the diagonalization of the Hamiltonian matrix. Such an approach has been employed in this work. Apart from ${ }^{3} \mathrm{He}$ and ${ }^{4} \mathrm{He}$ calculations, we also performed calculations for ${ }^{\infty} \mathrm{He}$ which are equivalent to calculations where the Born-Oppenheimer approximation is assumed.

After the wave functions were generated, expectation values of the internal relativistic operator have been calculated. The algorithms for the mass-velocity and Darwin terms have been derived and implemented in our previous works. $^{25-27}$ In this work we have derived and implemented the algorithms for calculating the orbit-orbit and spin-spin interactions. A detailed description of the algorithms will be presented elsewhere. ${ }^{31}$ One way those algorithms were tested was based on the Hermiticity of the orbit-orbit operator. Since this property is not as straightforward as for other relativistic operators, we included a short discussion on this point in the Appendix.

The results of the calculations that include all relativistic corrections of the order of $\alpha^{2}$ and the nonrelativistic and relativistic total energies are shown in Table I for ${ }^{3} \mathrm{He},{ }^{4} \mathrm{He}$, and ${ }^{\infty} \mathrm{He}$. The results for the ${ }^{4} \mathrm{He}$ ground state can be directly compared with the values obtained by Korobov and Yelkhovsky. ${ }^{2}$ Their ground state energy obtained with the nucleus-to-electron mass ratio of $7294.299508(16)$ 


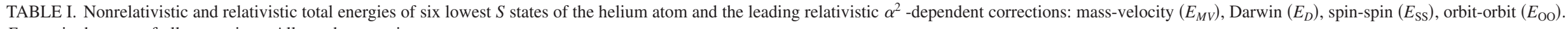
$E_{\text {tot.corr. }}$ is the sum of all corrections. All numbers are in a.u.

\begin{tabular}{|c|c|c|c|c|c|c|c|c|}
\hline State & Isotop & $E_{\text {nonrel }}$ & $E_{M V}$ & $E_{D}$ & $E_{\mathrm{SS}}$ & $E_{\mathrm{OO}}$ & $E_{\text {tot.corr. }}$ & $E_{\text {rel }}$ \\
\hline \multirow[t]{3}{*}{ 1S } & ${ }^{3} \mathrm{He}$ & -2.903167210710 & $-7.19524997 \times 10^{-4}$ & $5.87624314 \times 10^{-4}$ & $3.55647669 \times 10^{-5}$ & $-7.61606778 \times 10^{-6}$ & $-1.03951984 \times 10^{-4}$ & -2.903271162695 \\
\hline & ${ }^{4} \mathrm{He}$ & -2.903304557729 & $-7.19656687 \times 10^{-4}$ & $5.87704644 \times 10^{-4}$ & $3.55697376 \times 10^{-5}$ & $-7.56454048 \times 10^{-6}$ & $-1.03946845 \times 10^{-4}$ & -2.903408504575 \\
\hline & ${ }^{\infty} \mathrm{He}$ & -2.903724377034 & $-7.20059320 \times 10^{-4}$ & $5.87950288 \times 10^{-4}$ & $3.55849339 \times 10^{-5}$ & $-7.40698079 \times 10^{-6}$ & $-1.03931079 \times 10^{-4}$ & -2.903828808113 \\
\hline \multirow[t]{3}{*}{$2 S$} & ${ }^{3} \mathrm{He}$ & -2.145581923701 & $-5.46999721 \times 10^{-4}$ & $4.36436853 \times 10^{-4}$ & $2.89291421 \times 10^{-6}$ & $-6.52394403 \times 10^{-7}$ & $-1.08322349 \times 10^{-4}$ & -2.145690246049 \\
\hline & ${ }^{4} \mathrm{He}$ & -2.145678587578 & $-5.47098012 \times 10^{-4}$ & $4.36495589 \times 10^{-4}$ & $2.89327691 \times 10^{-6}$ & $-6.13047406 \times 10^{-7}$ & $-1.08322194 \times 10^{-4}$ & -2.145786909772 \\
\hline & ${ }^{\infty} \mathrm{He}$ & -2.145974046052 & $-5.47398524 \times 10^{-4}$ & $4.36675195 \times 10^{-4}$ & $2.89438585 \times 10^{-6}$ & $-4.92737345 \times 10^{-7}$ & $-1.08321681 \times 10^{-4}$ & -2.146082367733 \\
\hline \multirow[t]{3}{*}{$3 S$} & ${ }^{3} \mathrm{He}$ & -2.060896524119 & $-5.36331121 \times 10^{-4}$ & $4.28669362 \times 10^{-4}$ & $8.13908151 \times 10^{-7}$ & $-2.93108242 \times 10^{-7}$ & $-1.07140960 \times 10^{-4}$ & -2.061003665079 \\
\hline & ${ }^{4} \mathrm{He}$ & -2.060989082344 & $-5.36427390 \times 10^{-4}$ & $4.28727034 \times 10^{-4}$ & $8.14008624 \times 10^{-7}$ & $-2.54587012 \times 10^{-7}$ & $-1.07140934 \times 10^{-4}$ & -2.061096223278 \\
\hline & ${ }^{\infty} \mathrm{He}$ & -2.061271989736 & $-5.36721718 \times 10^{-4}$ & $4.28903387 \times 10^{-4}$ & $8.14315814 \times 10^{-7}$ & $-1.36802759 \times 10^{-7}$ & $-1.07140818 \times 10^{-4}$ & -2.061379130554 \\
\hline \multirow[t]{3}{*}{$4 S$} & ${ }^{3} \mathrm{He}$ & -2.033216570271 & $-5.33852455 \times 10^{-4}$ & $4.26939458 \times 10^{-4}$ & $3.34011039 \times 10^{-7}$ & $-2.11338835 \times 10^{-7}$ & $-1.06790324 \times 10^{-4}$ & -2.033323360595 \\
\hline & ${ }^{4} \mathrm{He}$ & -2.033307817477 & $-5.33948256 \times 10^{-4}$ & $4.26996896 \times 10^{-4}$ & $3.34051970 \times 10^{-7}$ & $-1.73013004 \times 10^{-7}$ & $-1.06790322 \times 10^{-4}$ & -2.033414607799 \\
\hline & ${ }^{\infty} \mathrm{He}$ & -2.033586717026 & $-5.34241156 \times 10^{-4}$ & $4.27172531 \times 10^{-4}$ & $3.34177116 \times 10^{-7}$ & $-5.58264836 \times 10^{-8}$ & $-1.06790275 \times 10^{-4}$ & -2.033693507301 \\
\hline \multirow[t]{3}{*}{$5 S$} & ${ }^{3} \mathrm{He}$ & -2.020809059294 & $-5.32990903 \times 10^{-4}$ & $4.26350646 \times 10^{-4}$ & $1.68027550 \times 10^{-7}$ & $-1.83245963 \times 10^{-7}$ & $-1.06655475 \times 10^{-4}$ & -2.020915714770 \\
\hline & ${ }^{4} \mathrm{He}$ & -2.020899726154 & $-5.33086543 \times 10^{-4}$ & $4.26408004 \times 10^{-4}$ & $1.68048051 \times 10^{-7}$ & $-1.44988870 \times 10^{-7}$ & $-1.06655479 \times 10^{-4}$ & -2.021006381633 \\
\hline & ${ }^{\infty} \mathrm{He}$ & -2.021176851555 & $-5.33378948 \times 10^{-4}$ & $4.26583397 \times 10^{-4}$ & $1.68110733 \times 10^{-7}$ & $-2.80126381 \times 10^{-8}$ & $-1.06655453 \times 10^{-4}$ & -2.021283507007 \\
\hline \multirow[t]{3}{*}{$6 S$} & ${ }^{3} \mathrm{He}$ & -2.014196551188 & $-5.32618704 \times 10^{-4}$ & $4.26098959 \times 10^{-4}$ & $9.61047987 \times 10^{-8}$ & $-1.71104194 \times 10^{-7}$ & $-1.06594744 \times 10^{-4}$ & -2.014303145932 \\
\hline & ${ }^{4} \mathrm{He}$ & -2.014286911171 & $-5.32714277 \times 10^{-4}$ & $4.26156286 \times 10^{-4}$ & $9.61164958 \times 10^{-8}$ & $-1.32877261 \times 10^{-7}$ & $-1.06594752 \times 10^{-4}$ & -2.014393505923 \\
\hline & ${ }^{\infty} \mathrm{He}$ & -2.014563098433 & $-5.33006479 \times 10^{-4}$ & $4.26331580 \times 10^{-4}$ & $9.61522589 \times 10^{-8}$ & $-1.59933099 \times 10^{-8}$ & $-1.06594739 \times 10^{-4}$ & -2.014669693173 \\
\hline
\end{tabular}


TABLE II. Nonrelativistic transition energies in comparison with relativistically corrected ones. All numbers are in megahertz.

\begin{tabular}{lrrrrr}
\hline \hline Transition & Isotope & $\Delta E_{\text {nonrel }}$ & $\Delta E_{\text {rel }}$ & Morton et al. (Ref. 1) & Expt. (Ref. 1) \\
\hline $2 S \rightarrow 1 S$ & ${ }^{3} \mathrm{He}$ & 4984671732 & 4984642976 & 4984604530 & 4984604593 \\
& ${ }^{4} \mathrm{He}$ & 4984939414 & 4984910625 & 4984872186 & 4984872191 \\
& ${ }^{\infty} \mathrm{He}$ & 4985757669 & 4985728780 & & \\
$3 S \rightarrow 2 S$ & ${ }^{3} \mathrm{He}$ & 557203162 & 557210935 & 557208896 & \\
& ${ }^{4} \mathrm{He}$ & 557230176 & 557237948 & 557235909 & 557235927 \\
& ${ }^{\infty} \mathrm{He}$ & 557312758 & 557320528 & & \\
$4 S \rightarrow 3 S$ & ${ }^{3} \mathrm{He}$ & 182125347 & 182127654 & 182127194 & \\
& ${ }^{4} \mathrm{He}$ & 182133973 & 182136280 & 182135821 & \\
& ${ }^{\infty} \mathrm{He}$ & 182160344 & 182162650 & & \\
$5 S \rightarrow 4 S$ & ${ }^{3} \mathrm{He}$ & 81637500 & 81638388 & 81638234 & \\
& ${ }^{4} \mathrm{He}$ & 81641319 & 81642206 & 81642053 & \\
& ${ }^{\infty} \mathrm{He}$ & 81652992 & 81653879 & & \\
$6 S \rightarrow 5 S$ & ${ }^{3} \mathrm{He}$ & 43508213 & 43508613 & 43508557 & \\
& ${ }^{4} \mathrm{He}$ & 43510232 & 43510632 & 43510576 & \\
& ${ }^{\infty} \mathrm{He}$ & 43516405 & 43516805 & & \\
\hline \hline
\end{tabular}

$$
E=-2.90330455772794023(1) \text { a.u. }
$$

is slightly higher than ours,

$$
E=-2.903304557729 \text { a.u. }
$$

Since we only used the double precision in the calculations, the number of significant figures reported in the energy is smaller than the number of significant figures in the results of Korobov and Yelkhovsky. In our calculations we used more current values of the physical constants and masses taken from CODATA 2002 Ref. 32 with the ${ }^{3} \mathrm{He}$ and ${ }^{4} \mathrm{He}$ nuclear masses equal to 5495.885269 and 7294.2995363 , respectively. Since our ${ }^{4} \mathrm{He}$ nuclear mass is slightly larger than that used by Korobov and Yelkhovsky, we recalculated the ${ }^{4} \mathrm{He}$ ground state energy using our basis set and their mass, and we obtained the value

$$
E=-2.90330455772757 \text { a.u., }
$$

which is slightly higher than their energy. A comparison can also be made between the relativistic correction of the order of $\alpha^{2}$ of Korobov and Yelkhovsky. They reported a value of $-1.95205077(1) \alpha^{2},{ }^{2}$ while our value is $-1.95200357 \alpha^{2}$.

Upon examining the results shown in Table I, one can notice that the mass-velocity, Darwin, and spin-spin relativistic corrections increase slightly in magnitude with the increase of the nuclear mass for all six $S$ Rydberg states calculated here. The increased mass of the nucleus leads to a slight spatial contraction of the system, higher average velocity of the electrons, and higher values of the electron-electron and electron-nucleus contact terms. This explains the increase of the mass-velocity, Darwin, and spin-spin relativistic corrections. This increasing trend is, however, not followed by the orbit-orbit correction. Here, not only does the correction decreases in magnitude with the rising mass of the nucleus, but this decrease also significantly accelerates for higher states. In those higher states, one of the electrons becomes excited to states that are increasingly more diffuse. When this hap- pens, the average distance of the excited electron from the nucleus significantly increases, and the radius of the motion of the nucleus around the center of mass of the system also increases. This effect is sensitive to the mass of the nuclei. For the infinite mass the radius of the motion of the nucleus around the center of mass becomes zero. This may explain the large difference between the orbit-orbit contribution for finite and infinite nuclear masses of the nucleus in higher Rydberg states.

Next, we used the calculated nonrelativistic and relativistic total energies of ${ }^{3} \mathrm{He},{ }^{4} \mathrm{He}$, and ${ }^{\infty} \mathrm{He}$ to determine the transition energies between the consecutive states. The transition energies are presented in Table II along with a comparison with the available experimental and calculated values obtained form ionization energies taken from the work of Morton et al. ${ }^{1}$ As mentioned before, those previously calculated values also include relativistic and QED corrections of higher orders in $\alpha$, and, due to that, are more accurate than our values.

\section{SUMMARY}

In this work we have shown that the gradient based variational optimization of explicitly correlated Gaussian functions leads to energies of ground and excited energies of the He atom, which agree very well with the best previously obtained values. The Gaussians seem to be capable of very well describing the oscillatory nature of the wave function which increases with the level of excitation. Unlike most of the previous atomic calculations, in the present approach we have not assumed the Born-Oppenheimer approximation regarding the separability of the electronic and nuclear motions. Thus, slightly different wave functions have been obtained for different $\mathrm{He}$ isotopomers. These functions have been used to calculate isotope-dependent relativistic corrections of the order of $\alpha^{2}$. 
Our future work will include extending the approach presented in this work to diatomic molecules and to states with a rotational quantum number higher than zero. Diatomic calculations will require the use of basis functions where the Gaussian exponents [Eq. (3)] are multiplied by powers of the internuclear distance.

\section{ACKNOWLEDGMENTS}

This work has been supported by the National Science Foundation. The authors would like to thank Professor Jacek Karwowski and Professor Lutoslaw Wolniewicz for many valuable discussions and suggestions concerning this work. Thanks are also due to Professor Jacek Komasa and Professor Krzysztof Pachucki for their valuable suggestions concerning evaluating some of the relativistic integrals.

\section{APPENDIX: HERMITICITY OF THE ORBIT-ORBIT OPERATOR}

In the laboratory Cartesian coordinate frame the orbitorbit operator has the following form:

$$
H_{\mathrm{OO}}=\frac{1}{2} \sum_{i=1}^{N} \sum_{j>i}^{N} \frac{Q_{i} Q_{j}}{M_{i} M_{j}}\left[\frac{1}{R_{i j}} \nabla_{i} \cdot \nabla_{j}+\frac{1}{R_{i j}^{3}} \mathbf{R}_{i j} \cdot\left(\mathbf{R}_{i j} \cdot \nabla_{i}\right) \nabla_{j}\right] .
$$

To simplify the problem let us consider a two-electron system where the orbit-orbit Hamiltonian is

$$
H_{\mathrm{OO}}=\frac{1}{2} \frac{e^{2}}{m_{e}^{2}}\left[\frac{1}{r_{12}} \nabla_{1} \cdot \nabla_{2}+\frac{1}{r_{12}^{3}} \mathbf{r}_{12} \cdot\left(\mathbf{r}_{12} \cdot \nabla_{1}\right) \nabla_{2}\right] .
$$

We will show that the Hermiticity of $H_{\mathrm{OO}}$ is a result of the cancellation of certain contributions resulting from the first part of the operator, $H_{1} \propto 1 / r_{12} \nabla_{1} \cdot \nabla_{2}$, and contributions resulting from its second part, $H_{2} \propto 1 / r_{12}^{3} \mathbf{r}_{12} \cdot\left(\mathbf{r}_{12} \cdot \nabla_{1}\right) \nabla_{2}$. We should note that $H_{1}$ and $H_{2}$ are not Hermitian by themselves. The Hermiticity of $H_{\mathrm{OO}}$ can be shown using the following commutational relations:

$$
\begin{aligned}
& {\left[\nabla_{2}, \frac{1}{r_{12}}\right]=-\left[\nabla_{1}, \frac{1}{r_{12}}\right]=\frac{\mathbf{r}_{12}}{r_{12}^{3}},} \\
& {\left[\nabla_{2}, \frac{\mathbf{r}_{12}}{r_{12}^{3}}\right]=-\left[\nabla_{1}, \frac{\mathbf{r}_{12}}{r_{12}^{3}}\right]=-4 \pi \delta^{3}\left(r_{12}\right),} \\
& {\left[\nabla_{2}, \mathbf{r}_{12}\right]=-\left[\nabla_{1}, \mathbf{r}_{12}\right]=-3,} \\
& {\left[\mathbf{A} \nabla_{2}, \mathbf{r}_{12}\right]=-\left[\mathbf{A} \nabla_{1}, \mathbf{r}_{12}\right]=-\mathbf{A},}
\end{aligned}
$$

where $\mathbf{A}$ is an arbitrary vector operator. Applying the above commutational relations to $H_{1}$ and $H_{2}$, we get

$$
\begin{aligned}
& {\left[\nabla_{1} \cdot \nabla_{2}, \frac{1}{r_{12}}\right]=\frac{\mathbf{r}_{12}}{r_{12}^{3}} \nabla_{1}-\frac{\mathbf{r}_{12}}{r_{12}^{3}} \nabla_{2}+4 \pi \delta^{3}\left(r_{12}\right),} \\
& {\left[\nabla_{1} \cdot \nabla_{2}, \frac{\mathbf{r}_{12}}{r_{12}^{3}} \mathbf{r}_{12}\right]=-\frac{\mathbf{r}_{12}}{r_{12}^{3}} \nabla_{1}+\frac{\mathbf{r}_{12}}{r_{12}^{3}} \nabla_{2}-4 \pi \delta^{3}\left(r_{12}\right)+a,}
\end{aligned}
$$

where $a$ is an operator whose expectation value is zero. Now, we add the two equations, [Eqs. (A1) and (A2)], and we get the following expression for the expectation value of the orbit-orbit interaction operator:

$$
\begin{aligned}
\left\langle\frac{1}{r_{12}}\right. & \left.\nabla_{1} \cdot \nabla_{2}\right\rangle+\left\langle\frac{\mathbf{r}_{12}}{r_{12}^{3}} \cdot\left(\mathbf{r}_{12} \cdot \nabla_{1}\right) \nabla_{2}\right\rangle \\
= & \left\langle\nabla_{1} \cdot \nabla_{2} \frac{1}{r_{12}}\right\rangle+\left\langle\frac{\mathbf{r}_{12}}{r_{12}^{3}} \cdot \nabla_{2}\right\rangle-\left\langle\frac{\mathbf{r}_{12}}{r_{12}^{3}} \cdot \nabla_{1}\right\rangle \\
& -4 \pi\left\langle\delta^{3}\left(r_{12}\right)\right\rangle+\left\langle\nabla_{1} \cdot\left(\nabla_{2} \cdot \frac{\mathbf{r}_{12}}{r_{12}^{3}}\right) \mathbf{r}_{12}\right\rangle \\
& -\left\langle\frac{\mathbf{r}_{12}}{r_{12}^{3}} \cdot \nabla_{1}\right\rangle+4 \pi\left\langle\delta^{3}\left(r_{12}\right)\right\rangle \\
= & \left\langle\nabla_{1} \cdot \nabla_{2} \frac{1}{r_{12}}\right\rangle+\left\langle\nabla_{1} \cdot\left(\nabla_{2} \cdot \frac{\mathbf{r}_{12}}{r_{12}^{3}}\right) \mathbf{r}_{12}\right\rangle,
\end{aligned}
$$

which clearly shows its Hermiticity.

${ }^{1}$ D. C. Morton, Q. Wu, and G. W. F. Drake, Can. J. Phys. 84, 83 (2006).

${ }^{2}$ V. I. Korobov and A. Yelkhovsky, Phys. Rev. Lett. 87, 193003 (2001).

${ }^{3}$ V. I. Korobov, Phys. Rev. A 66, 024501 (2002).

${ }^{4}$ K. Pachucki, Phys. Rev. Lett. 84, 4561 (2000).

${ }^{5}$ K. Pachucki, Phys. Rev. A 74, 022512 (2006).

${ }^{6}$ K. Pachucki, Phys. Rev. A 74, 062510 (2006).

${ }^{7}$ K. Pachucki and J. Sapirstein, J. Phys. B 35, 1783 (2002).

${ }^{8}$ G. W. F. Drake and S. P. Goldman, Can. J. Phys. 77, 835 (2000).

${ }^{9}$ A. Yelkhovsky, Phys. Rev. A 64, 062104 (2001).

${ }^{10}$ G. W. F. Drake and W. C. Martin, Can. J. Phys. 76, 679 (1998).

${ }^{11}$ D. Shiner, R. Dixson, and V. Vedentham, Phys. Rev. Lett. 74, 3553 (1995).

${ }^{12}$ G. W. F. Drake, W. Nördershäuser, and Z.-C. Yan, Can. J. Phys. 83, 311 (2005).

${ }^{13}$ D. M. From and R. N. Hill, Phys. Rev. A 36, 1013 (1987).

${ }^{14}$ F. E. Harris, A. M. Frolov, and V. H. Smith, J. Chem. Phys. 120, 9974 (2004).

${ }^{15}$ T. K. Rebane, V. S. Zotev, and O. N. Yusupov, Zh. Eksp. Teor. Fiz. 110, 55 (1996).

${ }^{16}$ V. S. Zotev and T. K. Rebane, Phys. Rev. A 65, 062501 (2002).

${ }^{17}$ K. Pachucki, M. Puchalski, and E. Remiddi, Phys. Rev. A 70, 032502 (2004).

${ }^{18}$ M. Puchalski and K. Pachucki, Phys. Rev. A 73, 022503 (2006).

${ }^{19}$ M. Cafiero, S. Bubin, and L. Adamowicz, Phys. Chem. Chem. Phys. 5, 1491 (2003).

${ }^{20}$ S. Bubin, M. Cafiero, and L. Adamowicz, Adv. Chem. Phys. 131, 377 (2005).

${ }^{21}$ D. B. Kinghorn and L. Adamowicz, J. Chem. Phys. 110, 7166 (1999).

${ }^{22}$ D. B. Kinghorn and L. Adamowicz, Phys. Rev. Lett. 83, 2541 (1999).

${ }^{23}$ S. Bubin and L. Adamowicz, J. Chem. Phys. 118, 3079 (2003).

${ }^{24}$ M. Pavanello, S. Bubin, M. Molski, and L. Adamowicz, J. Chem. Phys. 123, 104306 (2005).

${ }^{25}$ D. Kȩdziera, M. Stanke, S. Bubin, M. Barysz, and L. Adamowicz, J. Chem. Phys. 125, 084303 (2006).

${ }^{26}$ D. Kędziera, M. Stanke, S. Bubin, M. Barysz, and L. Adamowicz, J. Chem. Phys. 125, 014318 (2006).

${ }^{27}$ M. Stanke, D. Kẹdziera, M. Molski, S. Bubin, M. Barysz, and L. Adamowicz, Phys. Rev. Lett. 96, 233002 (2006).

${ }^{28}$ K. Pachucki and J. Komasa, Phys. Rev. A 73, 052502 (2006).

${ }^{29}$ K. Pachucki and J. Komasa, Phys. Rev. Lett. 92, 213001 (2004).

${ }^{30}$ K. Pachucki, W. Cencek, and J. Komasa, J. Chem. Phys. 122, 184101 (2005).

${ }^{31}$ S. Bubin, M. Stanke, D. Kẹdziera, and L. Adamowicz, Phys. Rev. A (to be published).

${ }^{32}$ See CODATA 2002 recommended values and NIST Physical Reference Data (http://physics.nist.gov/). 